\title{
Can infection after anterior cruciate ligament surgery be prevented by inserting a Hemovac drain into graft donor site?
}

\author{
Gökhan Bülent Sever, MD (1) \\ Department of Orthopedics and Traumatology, Gaziantep Private Sani Konukoğlu Hospital, Gaziantep, Turkey
}

Anterior cruciate ligament (ACL) reconstruction surgery is a highly successful orthopedic surgery providing both functional stability of the knee and more active participation of a person in daily life..$^{[1,2]}$ Postoperative infection following ACL reconstruction in normal population is reported to be highly rare with the incidence rate of only 0.14 to $1.4 \% .{ }^{[2-8]}$ On the other hand, the incidence rate among professional athletes was reported as high as 5.7\%. ${ }^{[9]}$ Although the occurrence of postoperative infection is very rare, once occurring, it causes devastating complications that can lead to significant problems such as cartilage destruction, arthrofibrosis, loss of range of motion and decreased activity level. ${ }^{[4,5,7,10,11]}$ Although there are no pre-defined treatment guidelines for the postoperative infection, the treatment of the infection after ACL reconstruction is open or arthroscopic debridement supported by usage of antibiotic. ${ }^{[12,13]}$ Preventing from infection becomes more critical because of both the difficulty of postoperative infection treatment and the high likelihood of adverse outcomes despite the extended treatment. Though previous studies have not revealed any exact cause, the frequency of infection in the use of hamstring autograft in ACL reconstruction surgery is higher than in bone patellar

Received: September 06, 2019

Accepted: October 08, 2019

Published online: March 02, 2020

Correspondence: Gökhan Bülent Sever, MD. Gaziantep Özel Sani Konukoğlu Hastanesi, Ortopedi ve Travmatoloji Bölümü 27090 Şehitkamil, Gaziantep, Türkiye.

E-mail: gokhanbsever@yahoo.com

Doi: $10.5606 /$ ehc. 2020.71334

Citation: Sever GB. Can infection after anterior cruciate ligament surgery be prevented by inserting a Hemovac drain into graft donor site?. Jt Dis Relat Surg 2020;31(1):143-148.

\section{ABSTRACT}

Objectives: This study aims to investigate the effect of Hemovac drainage placed in graft harvesting area on preventing deep surgical site infection (SSI) and/or septic arthritis through draining the hematoma formed in the graft area in arthroscopic primary anterior cruciate ligament (ACL) reconstruction surgery using hamstring autograft.

Patients and methods: This retrospective study was conducted between January 2008 and March 2019. A total of 819 patients (769 males, 50 females; mean age 33.7 years; range, 25 to 41 years) who underwent arthroscopic primary ACL reconstruction surgery using hamstring autograft were divided into two groups based on whether a Hemovac drain was also placed at the hamstring graft harvested area. Both groups were compared in terms of the presence of deep SSI and/or development of septic arthritis.

Results: In the non-drained group (group 1, n=401), 16 patients (3.9\%) had septic arthritis, four (0.9\%) had deep SSI, and two $(0.49 \%)$ had both wound and joint infections. Septic arthritis was identified in only one patient $(0.2 \%)$ in the drained group (group 2 , $\mathrm{n}=418$ ). In group 2, the mean amount of blood coming from the drain at the graft harvesting region was $36.85 \mathrm{~mL}$ (range, $20-50 \mathrm{~mL}$ ).

Conclusion: In arthroscopic primary ACL reconstruction surgery using hamstring tendon autograft, we concluded that the use of a Hemovac drain could be effective in preventing deep SSI through reducing the hematoma occurring in this region. However, future studies are needed to validate the effect of Hemovac drain on preventing the septic arthritis.

Keywords: Anterior cruciate ligament reconstruction, hamstring autograft, Hemovac drain, infection.

tendon bone autograft or allograft. ${ }^{[2,3,14]}$ Previously, Maletis et al. ${ }^{[2]}$ proposed some possible mechanisms in ACL reconstruction that lead to a high risk of infection in the hamstring autograft. These include (i) the length of time to prepare the hamstring graft, (ii) the likelihood of contamination, (iii) the proximity of the graft site to the tibial tunnel, and thus the possibility of transferring the infection within the joint, (iv) the presence of a hematoma in the graft site which creates 
an appropriate environment for bacteria placement, or $(v)$ the possibility of foreign body reaction of suture materials used in the preparation of the graft for hematoma. ${ }^{[2]}$ Infection after ACL reconstruction surgery used by the autograft can be seen in the graft, joint or both sites.

Since hematoma is a very suitable culture medium for the growth of bacteria, reducing the hematoma in the surgical field could lead to reducing the risk of infection. Accordingly, in this study, we aimed to investigate the effect of Hemovac drainage placed in graft harvesting area on preventing deep surgical site infection (SSI) and/or septic arthritis through draining the hematoma formed in the graft area in arthroscopic primary ACL reconstruction surgery using hamstring autograft.

\section{PATIENTS AND METHODS}

Between January 2008 and March 2019, a total of 920 patients who underwent arthroscopic ACL reconstruction surgery using hamstring tendon autograft in Orthopaedics and Traumatology Clinic of SANKO University Sani Konukoglu Research and Practice Hospital were analyzed retrospectively. Among these 920 patients, the patients who underwent a revision ACL reconstruction surgery and multiligamentous injuries $(n=65)$ were not included in the current study while other 36 patients could not be reached in postoperative follow-up. Thus, the current study included the analysis of a total number of 819 patients (769 males, 50 females; mean age 33.7 years; range, 25 to 41 years). We observed that all patients underwent primary arthroscopic ACL reconstruction with hamstring tendon autograft while two different fixation methods were used on the femoral side (EndoButton-CL [EndoButton Fixation Device, Smith Nephew, Memphis, USA] and Lift Systemed Ziploop [ToggleLoc Device with Ziploop Technology Zimmer Biomet, Biomet Orthopedics, Indiana USA]), and fixation was achieved with a metal cannulated capital letter " $K$ " screw on the tibial side. All surgeries were performed in the same surgical operating room, having a laminar airflow with a positive and HEPA filter keeping the room temperature constant between 18 and $20^{\circ} \mathrm{C}$. During the ACL surgery, two assistant nurses, one anesthesiologist, and one room staff assisted the surgeons in the operating room. A saline solution was used as a washing solution in the surgery. Patients were analyzed in terms of their demographic characteristics, infection predisposing diseases (e.g., diabetes mellitus), habits (e.g., smoking, alcohol use), hematoma amount from
Hemovac drain, and the presence of deep tissue infection and/or septic arthritis in the surgical field. The study protocol was approved by the SANKO University Ethics Committee for Clinical Research Trials (2019/02; 04.03.2019). A written informed consent was obtained from each patient. The study was conducted in accordance with the principles of the Declaration of Helsinki.

Intra-articular Hemovac drain was used in all patients routinely at the end of the surgery. In addition to this practice, after April 2013, Hemovac drain was also placed in the area where hamstring graft was harvested (Figure 1). The patients were divided into two groups based on whether the drain was placed in the region where hamstring autograft was harvested. In group 1, there was no usage of Hemovac drain in the harvesting area while in group 2, there was Hemovac drain usage in the harvesting area. These groups were then compared in terms of deep SSI and septic arthritis rates.

While harvesting the hamstring graft, a longitudinal incision was used over the adhesion of hamstring tendons just distal to the tibial tubercle.

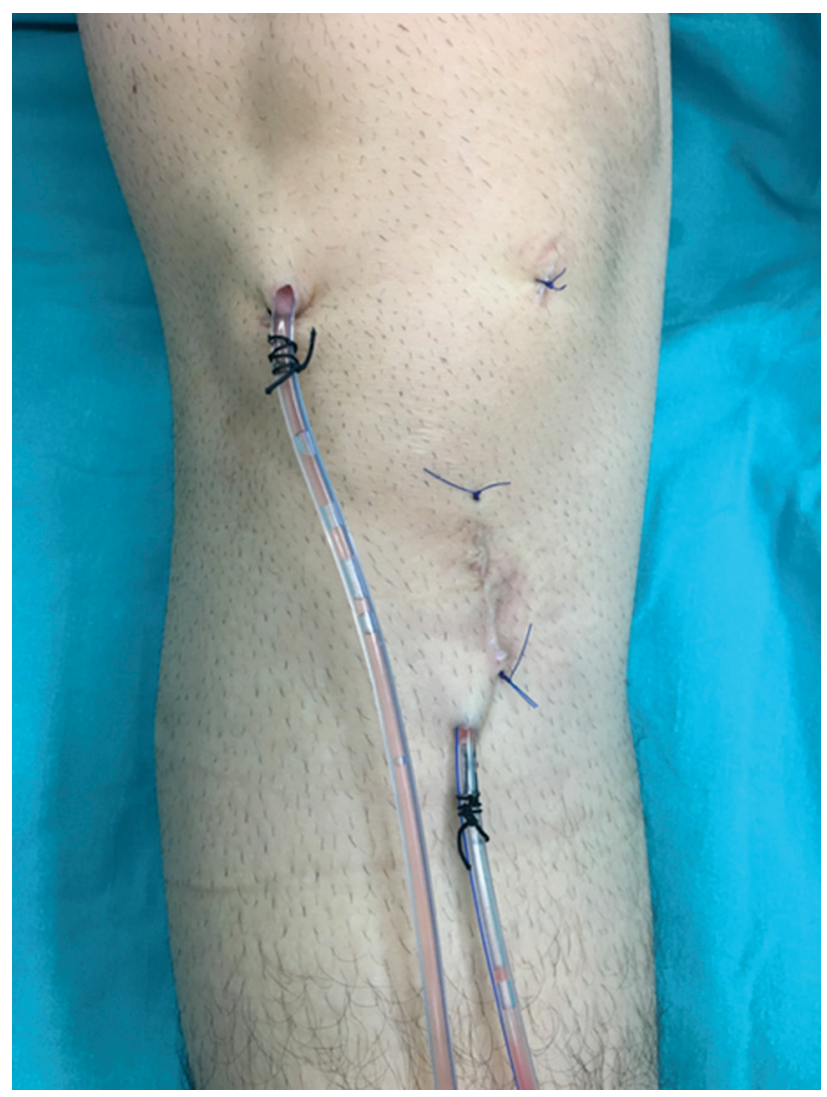

FIGURE 1. Hemovac drain placed in area where hamstring graft was harvested. 


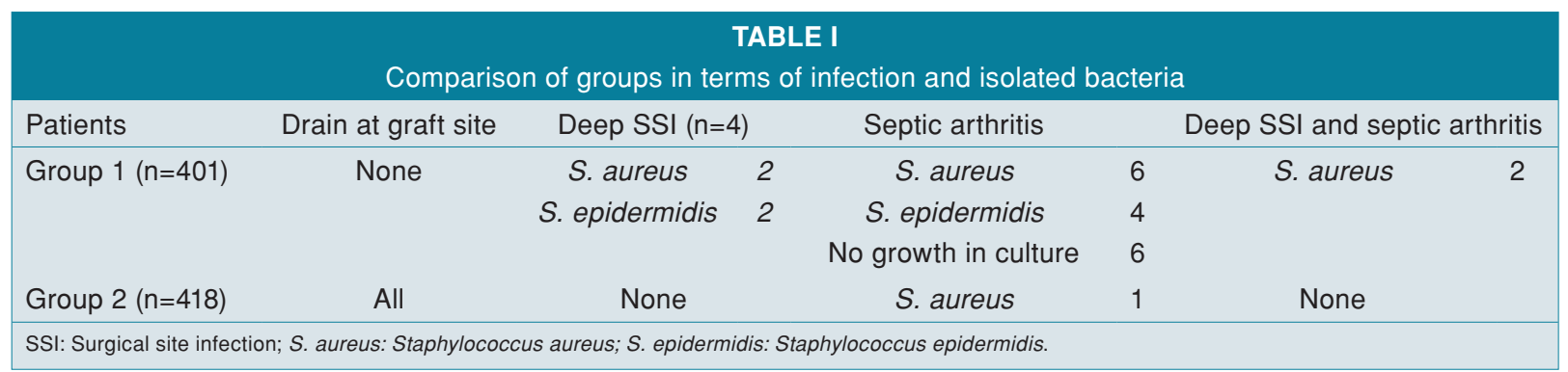

In group 2, the drain was extended to the proximal musculo-tendinous area where the tendon was harvested and fixed to the skin. Hemovac drains were removed at second postoperative day. Patients were followed-up at 3, 7, 15 days and 1, 2, 4 and 6 months postoperatively. The infection was identified during the follow-up after primary ACL reconstruction with local (incision line discharge, hyperemia, heat increase, knee effusion) and systemic clinical findings (systemic fever, nausea, vomiting, weakening) supported by laboratory findings (C-reactive protein and erythrocyte sedimentation rate).

\section{Statistical analysis}

Statistical analysis were carried out using SPSS version 15.0 software program (SPSS Inc., Chicago, IL, USA). Normal distribution of the continuous data was checked with Kolmogorov-Smirnov test. Independent groups t-test was used to compare two groups in terms of continuous data. Chi-square test was used to compare the qualitative data. Mean, standard deviation, percentage and frequency were reported as descriptive statistics. Significance level was set as $\mathrm{p}<0.05$.

\section{RESULTS}

Group 1 consisted of 401 patients (378 males, 23 females) who did not receive any drainage placement at the graft site. The mean age was 34.2 years in this group. Group 2 consisted of 418 patients (391 males, 27 females) whose Hemovac drain was placed in the graft region. The mean age was 33.7 years in group 2. There were no statistically significant differences between the two groups in terms of age and gender $(p=0.283$ and $p=0.722)$.

In group 2, the mean amount of blood coming from the drain at the graft harvesting region was $36.85 \mathrm{~mL}$ (range, 20-50 mL). During the follow-up, in group 1, septic arthritis was detected in 16 patients $(3.9 \%)$, deep SSI in four patients $(0.9 \%)$, and both wound and intra-articular infections were detected in two patients (0.49). In group 2, septic arthritis was detected in only one patient $(0.2 \%)$ (Table I). Infection predisposing chronic disease (e.g., diabetes mellitus) was not observed in any patient. In group 1, five patients were in the habit of smoking. Moreover, no predisposing habits were observed in any of the patients who developed infection.

In group 1, both of the two patients who developed extra- and intra-articular infections were found to have Staphylococcus aureus (S. aureus) in culture in both the graft and joint puncture specimens. S. aureus and coagulase (-) S. aureus were grown in two of the patients who developed deep SSI at the graft site. In 16 patients who developed septic arthritis, joint puncture fluid examination revealed $S$. aureus growth in six patients, Staphylococcus epidermidis growth in four patients while no culture growth was observed in six patients. In group 2, S. aureus was grown in the joint puncture fluid culture (Table I).

In group 1, arthroscopic washing, debridement of the surgical field and agent-directed antibiotherapy were used in two patients who developed the infection in both areas. In one patient, since septic arthritis and SSI developed again, intra-articular and surgical site debridement was required for the second time and grafts and implants were removed. The infection in this patient was controlled after this second surgery. In this group, the infection in the other four patients who developed infection only in the graft area was treated by surgical debridement and agent-directed antibiotic (teicoplanin). In 16 patients who developed septic arthritis, arthroscopic joint debridement and antibiotherapy were used initially. In three out of 16 patients, secondary washing was performed and grafts and implants were removed. In one of these three patients, $S$. aureus was grown while no growth in culture was observed in the other two patients. In the patient who developed septic arthritis in group 2, arthroscopic intra-articular washing, debridement and agent-directed antibiotic were used to control the infection. 


\section{DISCUSSION}

In this single-center retrospective study, we investigated the effect of Hemovac drainage in primary ACL reconstruction surgery using hamstring autograft on preventing the infection through draining the hematoma in this area. Our results showed that both septic arthritis and deep SSI were higher in the non-drained group $1^{[15]}$ In two patients $(0.49 \%)$ in whom no drainage was placed at the graft harvesting region, the same microorganism (S. aureus) was grown in both the surgical graft area and the intra-articular puncture fluid, indicating that the infection might be carried from the surgical site into the joint. While deep SSI occurred in six patients $(1.4 \%$, four in the surgical area and two in both the surgical area and the joint) in the non-drained group 1, no SSI was observed in group 2. The overall findings of this study suggest that draining the postoperative hematoma in the graft area is effective in preventing deep SSI, and its effect in preventing septic arthritis still needs to be supported by further multicenter studies.

Several previous studies have consistently reported that the infection formation after ACL reconstruction surgery is more frequently observed in patients using hamstring autograft. ${ }^{[2,3,14]}$ To prevent postoperative infection in ACL reconstruction surgery using hamstring autograft, researchers have suggested several approaches. For example, antibiotic prophylaxis or limitedly shaving the surgical area (only the required region for the surgical procedure) has been suggested in order to prevent orthopedic infections. ${ }^{[16,17]}$ To the best of our knowledge, our study is the first investigating the relationship between Hemovac drain and infection status. In patients who underwent ACL reconstruction surgery with hamstring autograft in our clinic until 2013, Hemovac drains were not placed in the graft harvesting area as a routine practice. Since the incidence of deep SSIs in our clinic was higher in the patient follow-up compared to the literature, we sought a solution to prevent the infection. The conditions of the operating room, sterilization of surgical instruments, sterilization of surgical clothes, shaving and cleaning of the surgical area, and prophylactic antibiotherapy procedure were all checked and no problems were detected. Thereafter, considering that the Hemovac drain placed in the graft site could be beneficial by removing the hematoma in this area, we have started to place Hemovac drain in the graft harvesting region as well. Since no infection was seen after the placing of the drains in graft harvesting region, we desired to share this improved outcome with the surgeons.
Since almost all infections occurred in the first six months postoperatively after ACL reconstruction surgery, ${ }^{[3,4,7,8,10]}$ we also reviewed the postoperative six-month period retrospectively in the current study. More specifically, we were interested in investigating the effects of reducing hematoma in the graft area to prevent deep surgical field and/or septic arthritis.

Postoperative hematoma creates a suitable environment for bacterial growth and predisposes to infection. ${ }^{[18]}$ Therefore, reducing hematoma in this area eliminates such appropriate environment for bacterial growth and offers the possibility to prevent infection. ${ }^{[19,20]}$ Draining the postoperative hematoma has been previously reported to be beneficial not only in reducing infection but also in preventing fibrosis. ${ }^{[21]}$ Several previous studies have suggested that the formation of hematoma increases the presence of prosthetic joint infection in patients using anticoagulants to prevent deep vein thrombosis after orthopedic surgery. ${ }^{[20,22,23]}$ For example, in their study on 972 primary total hip or total knee replacement cases, Lejitens et al. ${ }^{[20]}$ found that the infection was more frequent in the cases requiring high dose low molecular weight heparin prophylaxis. They further suggested that the higher frequency of this infection is associated with the excess amount of hematoma. ${ }^{[20]}$ Similarly, Burnett et al. ${ }^{[2]}$ also reported high rates of surgical site complications and infection in total hip or knee replacement surgeries in which they used standard dose low molecular weight heparin prophylaxis. ${ }^{[22]}$ Taking all these studies into consideration, it seems that reducing postoperative hematoma may reduce the postoperative infection rates. It can be further thought that the aforementioned studies were performed in arthroplasty patients and more hematoma formation may occur after such surgeries; therefore, infection rate may be increased. On the other hand, it is also suggested that reducing hematoma formation is effective in preventing infection in cases where less hematoma is formed in less invasive surgeries. Choi et al. ${ }^{[18]}$ examined 70 patients who underwent lumbar disc surgery and compared the cases with and without drainage in the operation area. They reported that infection was detected in two patients in the non-drainage group while they did not detect any infection in the drainage group. ${ }^{[18]}$ The mean amount of blood from the drain was reported to be $71 \mathrm{~mL}$ in the aforementioned study. In accordance with the above study, we found that the average blood flow from the drain was $36.85 \mathrm{~mL}$ in our study, and deep surgical field infection was observed in six $(1.43 \%)$ patients in the non-drained group 1, whereas 
no deep surgical field infection was observed in any of the drains. Septic arthritis was observed in 18 patients $(4.48 \%)$ in the non-drained group 1 and in one patient $(0.23 \%)$ in the drained group 2. In group 1 , two patients developed both deep SSI and septic arthritis. We observed that the same microorganism was grown in the culture of the samples taken from these areas, suggesting that deep SSI could be transferred into the knee via the tibial tunnel.

Closed system suction drainage is most commonly used to drain the hematoma in the surgical field. The effect of drainage use on preventing infection has been controversial. While some studies in the literature showed that the use of drainage does not affect the formation of infection, there have also been studies advocating that the use of drains increases the infection. ${ }^{[24-26]}$ For instance, Kanayama et al. ${ }^{[24]}$ reported that the rate of infection did not increase with the use of drainage in patients who underwent single-level lumbar decompression surgery whereas Zhou et al. ${ }^{[26]}$ reported that hemovac drain acts as a foreign body and increases surgical site infection.

This study has some limitations. The current study is a retrospective study and some changes in ACL reconstruction surgical techniques occurred during the study period. For example, for the preparation of the femoral tunnel in our clinic, we switched from transtibial technique to the technique using anteromedial portal. Moreover, EndoButton was previously used for femoral fixation in our clinic, and then we switched to cortical suspensory system. Furthermore, the surgical durations and infection frequencies of these fixation types were not evaluated during the study while the limited number of patients and the single-centered study design were the other limitations. Also, during the study period, while the operating room conditions did not change, the personnel changed and we could not investigate the possible effects of this situation on the infection rate. In addition, we could not compare the operative time of the two surgical techniques (EndoButton-CL and Lift Systemed Ziploop technique). Boddapati et al. ${ }^{[27]}$ reported that the hospital readmission percentage of patients who had a surgical operation longer than 90 minutes was higher in the first 30 days. Moreover, $25 \%$ of these patients admitted to the hospital for the second time due to infection. ${ }^{[27]}$ Increased surgical experience and shortening of the operation time may have been effective in decreasing the infection rate. However, the lack of any discussion on the effect of operative time on infection rate is another limitation of this study.
In conclusion, our overall findings suggest that the use of a Hemovac drain in ACL reconstruction surgery using hamstring tendon autograft is effective in preventing deep SSI through reducing the hematoma occurring in this region. However, the effect of Hemovac drain on preventing septic arthritis needs to be supported with future studies.

\section{Declaration of conflicting interests}

The author declared no conflicts of interest with respect to the authorship and/or publication of this article.

\section{Funding}

The author received no financial support for the research and/or authorship of this article.

\section{REFERENCES}

1. Atik OS. Is anterior cruciate ligament surgery protective against osteoarthritis? Eklem Hastalik Cerrahisi 2009;20:63.

2. Maletis GB, Inacio MC, Reynolds S, Desmond JL, Maletis MM, Funahashi TT. Incidence of postoperative anterior cruciate ligament reconstruction infections: graft choice makes a difference. Am J Sports Med 2013;41:1780-5.

3. Barker JU, Drakos MC, Maak TG, Warren RF, Williams RJ 3rd, Allen AA. Effect of graft selection on the incidence of postoperative infection in anterior cruciate ligament reconstruction. Am J Sports Med 2010;38:281-6.

4. Binnet MS, Başarir K. Risk and outcome of infection after different arthroscopic anterior cruciate ligament reconstruction techniques. Arthroscopy 2007;23:862-8.

5. Indelli PF, Dillingham M, Fanton G, Schurman DJ. Septic arthritis in postoperative anterior cruciate ligament reconstruction. Clin Orthop Relat Res 2002;398:182-8.

6. McAllister DR, Parker RD, Cooper AE, Recht MP, Abate J. Outcomes of postoperative septic arthritis after anterior cruciate ligament reconstruction. Am J Sports Med 1999;27:562-70.

7. Schollin-Borg M, Michaëlsson K, Rahme H. Presentation, outcome, and cause of septic arthritis after anterior cruciate ligament reconstruction: a case control study. Arthroscopy 2003;19:941-7.

8. Williams RJ 3rd, Laurencin CT, Warren RF, Speciale AC, Brause BD, O'Brien S. Septic arthritis after arthroscopic anterior cruciate ligament reconstruction. Diagnosis and management. Am J Sports Med 1997;25:261-7.

9. Sonnery-Cottet B, Archbold P, Zayni R, Bortolletto J, Thaunat M, Prost T, et al. Prevalence of septic arthritis after anterior cruciate ligament reconstruction among professional athletes. Am J Sports Med 2011;39:2371-6.

10. Judd D, Bottoni C, Kim D, Burke M, Hooker S. Infections following arthroscopic anterior cruciate ligament reconstruction. Arthroscopy 2006;22:375-84.

11. Schub DL, Schmitz LM, Sakamoto FA, Winalski CS, Parker RD. Long-term outcomes of postoperative septic arthritis after anterior cruciate ligament reconstruction. Am J Sports Med 2012;40:2764-70.

12. Mouzopoulos G, Fotopoulos VC, Tzurbakis M. Septic knee arthritis following ACL reconstruction: a systematic review. Knee Surg Sports Traumatol Arthrosc 2009;17:1033-42. 
13. Scully WF, Fisher SG, Parada SA, Arrington ED. Septic arthritis following anterior cruciate ligament reconstruction: a comprehensive review of the literature. J Surg Orthop Adv 2013;22:127-33.

14. Murphy MV, Du DT, Hua W, Cortez KJ, Butler MG, Davis RL, et al. Risk Factors for Surgical Site Infections Following Anterior Cruciate Ligament Reconstruction. Infect Control Hosp Epidemiol 2016;37:827-33.

15. Atik OŞ. Every new technique either conservative or surgical is good? Eklem Hastalik Cerrahisi 2019;30:183-4.

16. Tanner J, Norrie P, Melen K. Preoperative hair removal to reduce surgical site infection. Cochrane Database Syst Rev 2011;11:CD004122.

17. Trampuz A, Zimmerli W. Antimicrobial agents in orthopaedic surgery: Prophylaxis and treatment. Drugs 2006;66:1089-105.

18. Choi HS, Lee SG, Kim WK, Son S, Jeong TS. Is surgical drain useful for lumbar disc surgery? Korean J Spine 2016;13:20-3.

19. Alshryda S, Sarda P, Sukeik M, Nargol A, Blenkinsopp J, Mason JM. Tranexamic acid in total knee replacement: a systematic review and meta-analysis. J Bone Joint Surg [Br] 2011;93:1577-85.

20. Leijtens B, Kremers van de Hei K, Jansen J, Koëter S. High complication rate after total knee and hip replacement due to perioperative bridging of anticoagulant therapy based on the 2012 ACCP guideline. Arch Orthop Trauma Surg 2014;134:1335-41.

21. Payne DH, Fischgrund JS, Herkowitz HN, Barry RL, Kurz LT, Montgomery DM. Efficacy of closed wound suction drainage after single-level lumbar laminectomy. J Spinal Disord 1996;9:401-3.

22. Burnett RS, Clohisy JC, Wright RW, McDonald DJ, Shively RA, Givens SA, et al. Failure of the American College of Chest Physicians-1A protocol for lovenox in clinical outcomes for thromboembolic prophylaxis. J Arthroplasty 2007;22:317-24.

23. Tasker A, Harbord R, Bannister GC. Meta-analysis of low molecular weight heparin versus placebo in patients undergoing total hip replacement and post-operative morbidity and mortality since their introduction. Hip Int 2010;20:64-74.

24. Kanayama M, Oha F, Togawa D, Shigenobu K, Hashimoto $\mathrm{T}$. Is closed-suction drainage necessary for single-level lumbar decompression?: review of 560 cases. Clin Orthop Relat Res 2010;468:2690-4.

25. Poorman CE, Passias PG, Bianco KM, Boniello A, Yang S, Gerling MC. Effectiveness of postoperative wound drains in one- and two-level cervical spine fusions. Int J Spine Surg 2014;8. eCollection 2014.

26. Zhou XD, Li J, Xiong Y, Jiang LF, Li WJ, Wu LD. Do we really need closed-suction drainage in total hip arthroplasty? A meta-analysis. Int Orthop 2013;37:2109-18.

27. Boddapati V, Fu MC, Nwachukwu BU, Camp CL, Spiker AM, Williams RJ, et al. Procedure length is independently associated with overnight hospital stay and 30-day readmission following anterior cruciate ligament reconstruction. Knee Surg Sports Traumatol Arthrosc 2019. [Epub ahead of print] 